\title{
Open versus Laparoscopic Intraperitoneal Onlay Mesh for Repair of Spigelian Hernia: A Prospective Study
}

\author{
Mohammed Attia Elsayed, MD; Mohammed Shaaban Khalifa, MD; Wadie Boshra MD, MRCS \\ Department of General Surgery, Ain Shams University, Egypt
}

\begin{abstract}
Background: Is to describe the pathological, clinical and radiological features of Spigelian hernia, and to compare between open and laparoscopic methods of its surgical treatment.
\end{abstract}

Patients and methods: We describe the clinical management of 20 cases of Spigelian hernias as regard patient complaint, clinical examination, radiological investigations, and the surgical approach that was randomly performed either open or laparoscopic intraperitoneal only mesh.

Results: In all cases, the suspicion aroused during the clinical examination of the patients, and it was confirmed via imaging techniques. 10 cases were treated through open surgical approach, no intraoperative or postoperative complications have occurred, 3 patients had postoperative small wound seroma which resolved spontaneously, Other10 cases were treated through laparoscopic intraperitoneal onlay mesh repair with no intraoperative or postoperative complications. No recurrences had been observed for both groups in 24 months period of the study.

Conclusion: Spigelian hernia is a rare entity; its diagnosis is depending on a thorough clinical examination and must be confirmed by imaging studies especially CT scan which is the modality of choice for its diagnosis. Open surgical repair is considered the standard method for treatment due to its rarity but laparoscopic intraperitoneal onlay mesh repair is also safe and effective with shorter postoperative hospital stay.

Key words: Abdominal wall, ventral hernia, Spigelian hernia, laparoscopic intraperitoneal onlay mesh repair, surgery.

\section{Introduction}

Spigelian hernia is a rare entity of abdominal wall hernias with a reported incidence of $2 \%$. The clinical manifestation is usually non-specific and can be easily missed, a significant proportion of patients present with incarceration of Spigelian hernia $(\mathrm{SH})$ in their first visit. ${ }^{1}$

Spigelian hernia occurs through a slit like defects in the anterior abdominal wall adjacent to semilunar line, which extends from the tip of the ninth costal cartilage to the pubic spine at the lateral edge of rectus muscle inferiorly. Most of Spigelian hernias occur in the lower abdomen where the posterior sheath is deficient. The hernia ring is a well-defined defect in the transversus aponeurosis, the hernia sac is surrounded by extraperitoneal fatty tissue, and this sac is often interparietal passing through thetransversus and internal oblique aponeuroses and then spreading out beneath the intact aponeurosis of external oblique. ${ }^{2}$

Diagnosis of Spigelian hernia requires a high degree of suspicion, with the most common finding on clinical examination being a lump at the semilunar line. Radiological tests are useful in confirming the diagnosis. ${ }^{3}$

Surgical intervention is the gold standard for the management of $\mathrm{SH}$. The $\mathrm{SH}$ has been repaired by both conventional and laparoscopic approaches, Over the past decade there has been advancement in minimally invasive techniques for repair of $\mathrm{SH}$, with a reasonably good outcome. A number of approaches and techniques have been described. Yet, there was no agreed consensus on the best one. Although, open repair is the most common approach used, laparoscopic techniques have been increasingly used. It includes intraperitoneal onlay mesh (IPOM), transabdominal preperitoneal repair and totally extraperitoneal repair. ${ }^{4}$

\section{Patients and methods}

Demographic, clinical and surgical data of the twenty consecutive patients who presented with Spigelian hernia to surgical department in Ain Shams University hospitals (Cairo), Ibn Sina hospital (Jeddah) and El-Dar hospital (Medina) in the last 24 
months from July 2016 to July 2018 were reviewed. All clinical information was obtained from clinical records and referrals. All patients were subjected to imaging studies including abdominal ultrasound and Computed Tomography scan (CT scan) (Figure 2) to confirm the diagnosis and evaluate anatomical features like the size of the defect and the contents of the hernial sac.

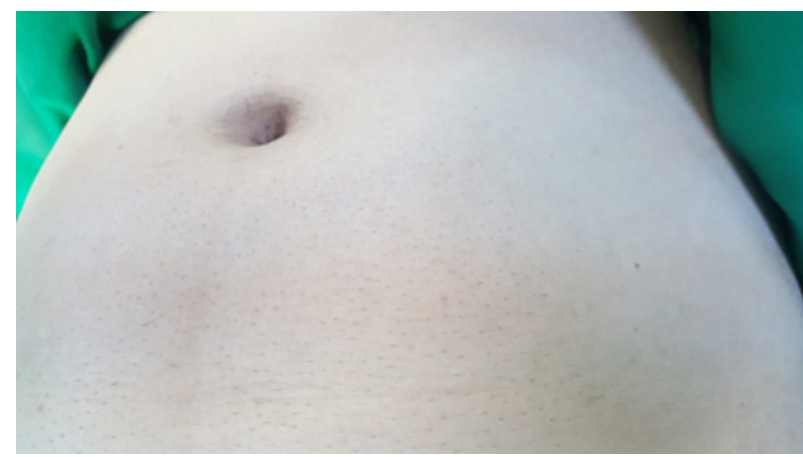

Fig 1: Clinically apparent Spigelian hernia on left side of abdomen.



Fig 2: CT image of anatomical defect in SH.

All patients were evaluated by routine blood investigation, chest $\mathrm{x}$-ray and ECG. All patients signed preoperative written informed consent. Then patients were randomly classified into 2 groups. The patients of the 1 st group (10 patients) underwent open surgery with the use of prosthetic mesh placed on the sheath, while the patients of the 2 nd group (10 patients) underwent laparoscopic intraperitoneal onlay mesh repair with the use of double layer composite mesh.

\section{Operative techniques: \\ Open Surgical Approach}

All operations were conducted in supine position undergeneral anesthesia, transverse incision was sited over the protrusion, and the external oblique aponeurosis was incised in the direction of fibers to expose the peritoneal sac. In most cases, the sac was inverted but in some cases the hernial sac was opened and the adhesions were sectioned then hernial orifice was closed with nonabsorbable sutures and reinforced via a prosthetic polypropylene mesh placed on sheath, in all cases, the contents were viable with surgical drain sited over the mesh.

\section{Laparoscopic intraperitoneal onlay mesh repair}

Intraperitoneal access was performed using Veress needle. Once abdominal access was obtained, site of hernial orifice was readily identified and ports were placed at least $10 \mathrm{~cm}$ away from the hernial defect in the form of an arc of a circle whose center is the hernial defect. Contents were reduced from the sac and adhesiolysis was performed if required to free 5 $\mathrm{cm}$ around the defect for a double layer composite mesh (Symbotex, Autosuture; Covidien, Mansfield, Massachusetts, USA) to be placed. The mesh was fixed using a combination of transabdominal sutures and tacks (Autosuture, Tyco health care, US surgicals, CT, USA). The mesh was adjusted to cover 3-5 cm all around the defect. Two patient had coexistent paraumbilical hernia that was repaired at the same session.

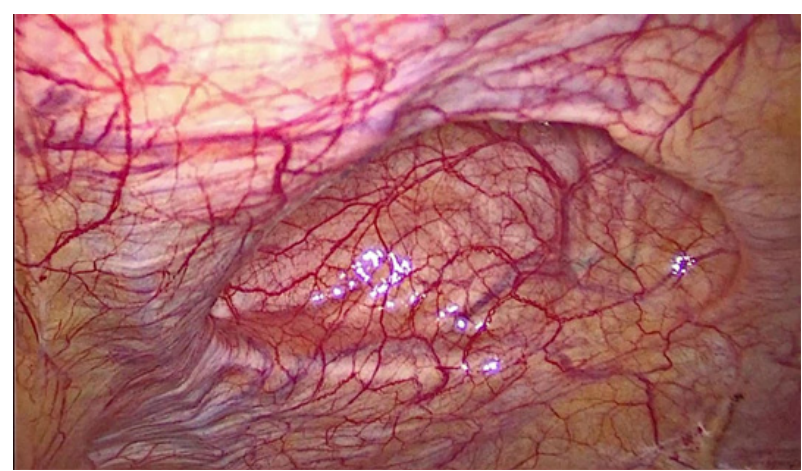

Fig 3: Laparoscopic view of the defect after reduction of the contents.

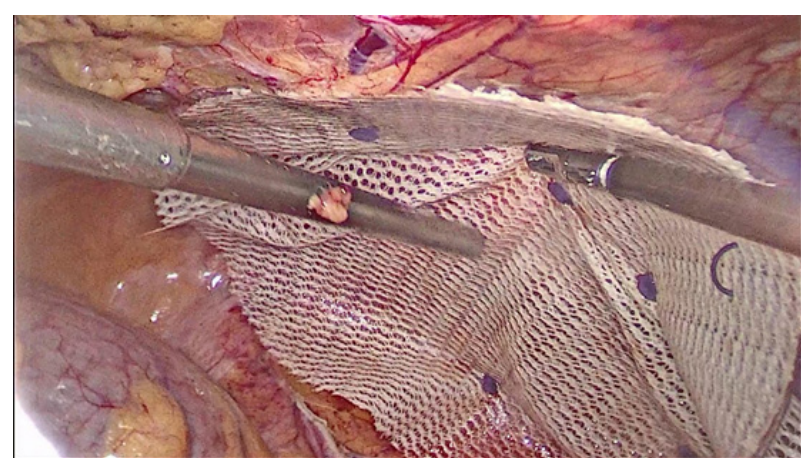

Fig 4: Laparoscopic view showing the mesh covering the defect during fixation with tackers. 
Then all patients of both groups were instructed to come for regular follow up every 3 months where adequate history was taken and good clinical examination was done in each visit, also abdominal ultrasound and / or CT scan was done if required to exclude suspected recurrence.

All operative and postoperative data of the patients of both groups were collected and analyzed.

Then both groups were compared as regard to the duration of surgery, intraoperative and postoperative complications, length of hospital stay, number of cases diagnosed by US and CT scan and recurrence rate.

\section{Statistical analysis}

Data were collected, revised, coded and entered to the Statistical Package for Social Science (IBM SPSS) version 23. Data were presented as percentages. The differences in surgical outcomes between the 2 groups were compared using the Pearson $X 2$ and Fisher exact tests. $\mathrm{P}$ values were reported where the results were considered to be significant (S) with $\mathrm{p}<0.05$, highly significant with $\mathrm{p}<0.01$ and non significant (NS) with $\mathrm{p}>0.05$.

\section{Results}

In our study, the mean age of the ten patients that were operated upon by open surgical technique was 56 y (40-72). Of these ten patients, 7 were females $(70 \%)$ and 3 were males $(30 \%)$, while the mean age of the ten patients that were operated upon by laparoscopic intraperitoneal onlay mesh repair was $54 y$ (38-70). Of these 10 patients 6 were females $(60 \%)$ and 4 were males (40\%).

For the patients of open group the hernias were located in the left side in 6 patients $(60 \%)$ and in the right side in 4 patients $(40 \%)$ while for the laparoscopic group 8 hernias (80\%) were located in the left side and 2 hernias (20\%) were located in the right side.

As regard to the patients of the open group 6 patients (60\%) presented by a palpable abdominal lump (Figure 1), 2 patients (20\%) presented by abdominal pain related to the site of hernia and 2 patients (20\%) presented by palpable abdominal lump together with pain . As regard to the patients of the laparoscopic group 5 patients (50\%) presented by palpable abdominal lump, 2 patients (20\%) were presented by abdominal pain and 3 patients $(30 \%)$ presented by palpable abdominal lump together with pain. The mean period of symptoms was $3 y$
$(2-5)$ years for the patients of open group and $5 y$ (3-8) years for the patients of laparoscopic group.

As regard to the open group 4 patients (40\%) were hypertensive on medical treatment, 2patient (20\%) were diabetic on regular insulin, 2 patients $(20 \%)$ were hypertensive and diabetic on regular treatment and 3cases (30\%) were obese with BMI $>40$, while for the laparoscopic group 2 patients(20\%) were hypertensive on medical treatment, 3 patients(30\%) were diabetic one on regular insulin and other on oral hypoglycemics and 4 patients $(40 \%)$ were obese with BMI $>40$. all patients of both groups were controlled preoperative on medical treatment. The preoperative diagnosis was done clinically in 9 patients (90\%) of the open group, and in 8 patients $(80 \%)$ of the laparoscopic group. The diagnosis was confirmed with abdominal ultrasound (US) in 8 cases (80\%) of the open group and in 7 cases $(70 \%)$ of the laparoscopic group, diagnosis further confirmed via abdominal computed tomography scan (CT scan) (Figure 2) in all 10 cases (100\%) in the open group and in 9 cases (90\%) of the laparoscopic group. The mean defect diameter was found to be 6(3-12) cm for the open group and 4(39) $\mathrm{cm}$ for the laparoscopic group.

The average range of operating time was 47 minutes (29-70 minutes) for the open group and 42 minutes (30-56 minutes) for the laparoscopic group. Some patients had co existent other abdominal wall hernias, as regard to open surgical group two patients had left sided inguinal hernia, one had left sided femoral hernias, all hernias were operated in the same session together with Spigelian hernia repair. As regard to laparoscopic group two patients had coexistent paraumbilical hernia that was repaired at the same session. The mean length of postoperative hospital stay was 4 days (2-7) days for the open group versus 1.3days (1-2 days). No intraoperative complications were encountered in both groups.

Only 3cases (30\%) of the open surgical group developed postoperative complications in the form of wound seroma that were controllable and treated conservatively but no postoperative complications noted in the laparoscopic group. The follow up period was found to be 8 to 22 months (average 17 months). follow up was done in outpatient clinic for all patients of both groups that had regular clinical assessment; no recurrence was detected in both groups with satisfactory postoperative outcome. 
Table 1: Demographic, clinical, radiological and operative data of patients of each group

\begin{tabular}{|c|c|c|c|}
\hline Variable & Open Approach $(n=10)$ & $\begin{array}{l}\text { Laparoscopic intraperitoneal } \\
\text { onlay mesh }(n=10)\end{array}$ & P Value \\
\hline Mean age & 56 y $(40-72)$ & 54 y $(38-70)$ & 0.42 (NS) \\
\hline Gender (male/female) & $3 / 7$ & $4 / 6$ & 0.59 (NS) \\
\hline Side of hernia (Rt/Lt) & $4 / 6$ & $2 / 8$ & 0.09 (NS) \\
\hline \multicolumn{4}{|l|}{ Patient presentations: } \\
\hline Palpable abdominal lump & $6(60 \%)$ & $5(50 \%)$ & \multirow{3}{*}{0.9 ( NS) } \\
\hline Abdominal pain & $2(20 \%)$ & $2(20 \%)$ & \\
\hline Palpable abdominal lump with pain & $2(20 \%)$ & $3(30 \%)$ & \\
\hline Mean period of symptoms & $3 y(2-5)$ & $5 y(3-8)$ & $0.112(\mathrm{NS})$ \\
\hline Mean defect diameter & $6(3-12) \mathrm{cm}$ & $4(3-9) \mathrm{cm}$ & 0.075 (NS) \\
\hline Mean follow up period & 17 months & 17 months & \\
\hline \multicolumn{4}{|l|}{ Associated co morbidity } \\
\hline Hypertension & $4(40 \%)$ & $2(20 \%)$ & \multirow{4}{*}{$0.8(\mathrm{NS})$} \\
\hline Diabetes Mellitus & $2(20 \%)$ & $3(30 \%)$ & \\
\hline Hypertension with Diabetes & $2(20 \%)$ & 0 & \\
\hline Obesity & $3(30 \%)$ & $4(40 \%)$ & \\
\hline \multicolumn{4}{|l|}{ Sensitivity of Diagnostic modalities: } \\
\hline Abdominal US & 8 cases $(80 \%)$ & 7 cases $(70 \%)$ & \multirow[t]{2}{*}{0.08 (NS) } \\
\hline Abdominal CT scan & 10 cases $(100 \%)$ & 9 cases $(90 \%)$ & \\
\hline Other coexistent hernia & 2 cases $(20 \%)$ & 2 case $(20 \%)$ & $>0.05$ (NS) \\
\hline Average duration of operation(min) & $47(29-70)$ & $42(30-56)$ & 0.07 (NS) \\
\hline Intraoperative complications & 0 & 0 & NS \\
\hline Postoperative complications & $3(30 \%)$ & 0 & $<0.05(S)$ \\
\hline $\begin{array}{l}\text { Average length of postoperative hospi- } \\
\text { tal stay (days) }\end{array}$ & $4(2-7)$ & $1.3(1-2)$ & $<0.01(\mathrm{HS})$ \\
\hline Recurrence & 0 & 0 & NS \\
\hline
\end{tabular}

\section{Discussion}

Spigelian hernia is named after (ArianeVanSpigel) ${ }^{5}$ who described the semilunar line, whoever the hernia was first described by (KlinKosch) in 1764. The hernia appears to peak in the 4th to7th decades, the male to female ratio is $1: 1.8$. Spigelian hernias are very uncommon and constitute only $0.12 \%$ of all abdominal wall hernias. ${ }^{6}$ Spigelian hernia can be congenital or acquired. The Spigelian aponeurosis is widest between $0-6 \mathrm{~cm}$ cranial to the interspinous plane and $85-90 \%$ of the hernias occur within the Spigelian hernia belt. ${ }^{7}$

Spigelian hernias represent between 0.1 and $2 \%$ of all abdominal wall hernias and are more frequent in older people (40-70 year old), especially in women, and are very occasionally bilateral. ${ }^{8}$ In our study the mean age for the open group was 56 years and was 54 years for the laparoscopic group. Female cases were 7 of $10(70 \%)$ of open group and were 6 of 10 $(60 \%)$ of laparoscopic group. All these reports were consistent with other series which stated that $\mathrm{SH}$ is most commonly seen in the middle age group and more frequently in females with a ratio of $1.4: 1 .{ }^{9}$

The diagnosis of Spigelian hernia is difficult and few surgeons suspect it, it has no characteristics symptoms and the hernia may be interparietal with no obvious mass on inspection or palpation. Only $50 \%$ of cases are diagnosed preoperatively. ${ }^{10}$

In our study the preoperative diagnosis was done clinically in 9 patients ( $90 \%)$ of the open group, and in 8 patients $(80 \%)$ of the laparoscopic group. As regard, the patients of the open group; 6patients $(60 \%)$ presented by a palpable abdominal lump, 2 patients (20\%) presented by abdominal pain related to the site of hernia and 2 patients (20\%) presented by palpable abdominal lump together with pain. As regard to the patients of the Laparoscopic group 5 patients $(50 \%)$ presented by palpable abdominal lump, 2 patients (20\%) presented by abdominal pain and 3 patients $(30 \%)$ presented by palpable abdominal lump together with pain. These figures were comparable with the results of a retrospective study done by (Larson and Farley) ${ }^{11}$ at Mayo clinic 
that documented that $35 \%$ of their patients had a palpable swelling, $24 \%$ had abdominal pain, and only $6 \%$ were completely asymptomatic.

Ultrasound is recommended as first line imaging investigation, with this aid, the correct diagnosis was obtained in 8 of 10 patients $(80 \%)$ of the open group and in 7 of 10 patients (70\%) of the laparoscopic group were studied. The advantages of real time ultrasonography are the ability to perform examination in both supine and up right positions, and while the patient performs a Valsalva maneuver. ${ }^{12}$ CT scan with close thin sections is considered the most reliable technique to make the diagnosis in doubtful cases. The use of oral contrast medium during the examination is recommended, so that any bowel content can be identified. ${ }^{13} \mathrm{CT}$ scan confirms the diagnosis in 10 of 10 patients $(100 \%)$ of the open surgical group and in 9 of 10 patients (90\%) of laparoscopic group which indicates much higher sensitivity of CT scan in comparison to ultrasound in diagnosing Spigelian hernia. These results were comparable with other series. ${ }^{14}$ In another study CT is reported to have a sensitivity of $100 \%$ and a positive predictive value (PPV) of $100 \%$, whereas ultrasonography has a sensitivity of $90 \%$ and a PPV of $100 \%$, and clinical assessment alone has a sensitivity of $100 \%$ and a PPV of $36 \% . .^{15}$ The differential diagnosis includes appendicitis and appendicular abscess, abdominal wall tumors or spontaneous hematoma of rectus sheath or even acute diverticulitis. ${ }^{16}$

As regard location of hernia, 6 patients (60\%) of the open group had $\mathrm{SH}$ on left side, which is more frequent as compared to contra lateral side, while 4 patients $(40 \%)$ had hernia on right side, while for the laparoscopic group 8 patients had left sided hernia and two patients had right sided hernia. as for the etiology the majority of cases were acquired hernias. because the Spigelian fascia was among the less resistant areas of the abdominal wall. ${ }^{17}$

Other abdominal wall hernias are not uncommon associations with $\mathrm{SH}$ which may suggest a predisposition to abdominal wall defects in patients with $\mathrm{SH}^{18}$ In our study there were 2 cases (20\%) with another hernia associating with $\mathrm{SH}$ (one with left inguinal hernia and other with left femoral hernia) among the open surgical group while in the laparoscopic group there was 2 cases (20\%) with paraumbilical hernia associating with $\mathrm{SH}$. These reports were consistent with several case reports that have reported simultaneous repair of other hernias such as umbilical ${ }^{19}$ and inguinal hernias. ${ }^{20}$

The treatment of Spigelian hernia is essentially surgical, and due to its high incidence of complications (35\% strangulations, incarceration), surgery is recommended in almost all cases. ${ }^{21}$ The optimal surgical approach for repair is not yet well defined, as surgeon can use a traditional open approach or a laparoscopic technique. ${ }^{22}$

(Carter and Mizes) ${ }^{23}$ treated $\mathrm{SH}$ with laparoscopic approach for the first time in 1992, the laparoscopic repair can be performed through 2 different accesses, a trans abdominal and extraperitoneal, the latter was published for the first time by (Moreno-Egea et al) in 1999, whoever, the intraperitoneal technique is considered now the best current technique for laparoscopic repair of $\mathrm{SH} .{ }^{24}$

The laparoscopic technique is constantly evolving and becomes today the gold standard approach for non-complicated cases in referral centers, is still requiring a good learning curve which may requires several years of practice, given the rarity of disease, for these reasons together with high incidence of complications, the open surgical repair remain the recommended approach. ${ }^{11}$

In our study there were 10 patients who underwent open approach versus 10 patients who underwent laparoscopic intraperitoneal onlay mesh. The comparison of both groups reflected that there were no significant differences in demographic, clinical and radiological features of the patients of both groups; also no significant difference in the average operating time was noted between both groups, but there were significant differences as regard to the average length of postoperative hospital stay which was shorter as regard to the laparoscopic group (1.3 vs. 4 days). This feature was nearly consistent with a randomized trial done by (Moreno-Egea et al) ${ }^{13}$ comparing open versus laparoscopic repair of $\mathrm{SH}$ in the literature concluded that the laparoscopic group significantly reduced the morbidity of patients and the length of hospital stay. Some authors also advocate for laparoscopic repair to avoid disrupting the physiological behavior and mechanics of abdominal wall through scarring in open surgery. ${ }^{25}$

From the review of the literature, the overall recurrence rate of $\mathrm{SH}$ is varies and ranges from 5 to $14 \% .{ }^{26}$ In our study there was no recurrence in both groups over a period of 24 months follow up. In a series of 70 patients undergoing open primary suture repair, there were 3 recurrences (4.3\%) which subsequently underwent further repair with no further recurrence. ${ }^{11}$

\section{Conclusion}

Spigelian hernia is a rare condition with limited number of cases published in the scientific literatures. Diagnosis of Spigelian hernia needs thorough clinical examination with high suspect and needs to be confirmed by imaging techniques especially CT scan. Open surgical repair remains the 
traditional treatment strategy especially in urgent cases but laparoscopic intraperitoneal onlay mesh repair is also safe and effective with shorter hospital stay and less postoperative morbidity.

\section{References}

1. Spangen L: Spigelian hernia. World J Surg. 1999; 13: 573-580.

2. Spangen L: Spigelian hernia. Surg. Clin North Am. 1997; 64: 351-366.

3. Zaki W, Alawad AAM: Spigelian Hernia: A rare case report. Ann Clin Case Rep. 2017; 2: 1288.

4. Srivastava KN, Agarwal A: Spigelian hernia. A diagnostic dilemma and laparoscopic management. Indian J Surg. 2015; 77(Suppl 1): S35-S37.

5. Engeset J, Youngson GC: Ambulatory peritoneal dialysis and hernia complications. Surg Clin North Am. 2007; 64: 385-392.

6. Opson RD, Davis WC: Spigelian hernia: Rare or obscure? Am J Surg. 2009; 116: 842-846.

7. Read RC: Observation on the etiology of Spigelian hernia. Ann Surg. 2012; 152: 10041009.

8. Mouton WG, Otten KT, Weiss D, Naef M, Wagner HF: Preperitoneal mesh repair in Spigelian hernia. Int Surg. 2006; 91: 262-264.

9. Polistina FA, Garbo G, Trevisan P, Frego M: Twelve years of experience treating Spigelian hernia. Surgery. 2015; 157: 547-550.

10. Campos SM, Walden T: Images in clinical medicine: Spigelian hernia. $\boldsymbol{N}$ Eng $\boldsymbol{J}$ Med. 2008; 336: 1149.

11. Larson DW, Farley DR: Spigelian hernias; repair and outcome for 81 patients. World J Surg. 2002; 26: 1277-1281.

12. Rogers FB, Camp PC: A strangulated Spigelian hernia mimicking diverticulitis. Hernia. 2014; 5: 51-52.

13. Moreno-Egea A, Carrosco L, Girela E, et al: Open vs. laparoscopic repair of Spigelian hernia: A prospective randomized trial. Arch Surg. 2002; 137: $1266-1268$.

14. Webber V, Low C, Skipworth RJ, Kumar S, de Beaux AC, Tulloh B: Contemporary thoughts on the management of Spigelian hernia. Hernia. 2017.
15. Light D, Chattopadhyay D, Bawa S: Radiological and clinical examination in the diagnosis of Spigelian hernias. Ann R Coll Surg Engl. 2013; 95: 98-100.

16. Vesico G, Sommella L, Gallelli G: Complicated Spigelian hernia: Our experience. Ann Ital Chir. 2011; 71: 573-578.

17. Zennaro F, Tasi D, Orio A, Morelli C, Chella B: Spigelian hernia. Anatomico-clinical considerations and description of 5 clinical cases. Ann Ital Chir. 2012; 74: 165-168.

18. Paliogiannis P, Delgou L, Contu G, et al: Small bowel emergencies: "Two surgical center" experience and literature review. Eur Surg. 2016: 48: 18-25.

19. Amendolara M: Video laparoscopic treatment of Spigelian hernias. Surg Laparosc Endosc. 1998; 8: 136-139.

20. Vannahme $M$, Monkhouse SJ: Acute management of a unilateral incarcerated Spigelian hernia in a patient with bilateral Spigelian hernias. Ann R Coll Surg Engl. 2013; 95: e89-91.

21. Mittal $T$, Kumar $V$, Khullar $R$, et al: Diagnosis and management of Spigelian hernia: A review of literature and our experience. $\boldsymbol{J}$ Min Access Surg. 2014; 4:95-98.

22. Carter JE, Mizes C: Laparoscopic diagnosis and repair of Spigelian hernia: report of a case and technique. Am J Obstet Gynecol. 1992; 167: 77-78.

23. Perrakis $A$, Velimezis $G$, Kapogiantos $G m$, et al: Spigelian hernia: A single center experience in a rare hernia entity. Hernia. 2015; 16: 439-445.

24. Conzo G, Giordano A, Candela G, et al: Giant Spigelian hernia associated with inguinal hernia. Repair with poly propylene prosthesis. $\mathbf{A m} \boldsymbol{J}$ Surg. 2015; 55: 611-615.

25. Leff DR, Hassell J, Sufi P, Heath D: Emergency and elective laparoscopic repair of spigelian hernias; two case reports and a review of the literature. Surg Laparosc Endosc Percutan Tech. 2009; 19: e152-155.

26. Moreno-Egea A, Campillo-Soto A, MoralesCuenca G: Which should be the gold standard laparoscopic technique for handling Spigelian hernias? Surg Endosc. 2015; 29: 856-862. 